\title{
CARDIAC MANIFESTATIONS IN A CASE OF TUBEROUS SCLEROSIS
}

\author{
BY \\ F. P. DURAS \\ From St. Andrew's Hospital, Northampton \\ Received October 12, 1944
}

The case reported in this communication is alive and relatively well at the time of writing. A rhabdomyoma of the heart is strongly suspected, though the diagnosis can be made with certainty only after death.

\section{CASE REPORT}

The patient, a young man of 23, was first seen in June, 1942. He was then a high-grade mental defective with a limited vocabulary, though he could give a fairly clear account of his life, and his memory was fairly good. He could read and write, and had a number of interests, such as listening to the wireless, keeping a daily record of the outside temperature, etc. When seen last (June, 1944), he was sprightly, impish, mischievous, and full of tricks, and always highly amused afterwards when taken seriously; he had the outlook of a boy of seven. He was the only child of healthy parents, and there was no mental illness on the paternal or maternal side of the family. Birth and pregnancy were normal, but he was a fretful baby and only began to walk when two years old and to talk at the age of three. He tried school but failed. In 1934 he was incontinent for urine, mostly by day, and passed water very frequently. The patient himself reported that he had attacks of dizziness and falling down, and that he " goes to sleep " for a few minutes sometimes whilst standing, but this was not observed by the parents. He had no epileptic fits while under medical observation for the last two years, but the above-mentioned "turns" might be regarded as epileptic equivalents.

Physical examination showed a youth of asthenic build, $5 \mathrm{ft}$. 6 in. in height, of poor muscular development, weighing 8 stone 12 pounds. The nose and cheeks were conspicuously covered with sebaceous adenoma in the form of reddish brown nodules of pinhead size, distributed in the classical " butterfly" pattern. The skin of the upper part of the chest had a gooseflesh texture; on the back of the neck were numerous short tags of skin sticking out like bristles, whilst on the back itself there were, especially on its lower part, a few irregular, thickened, whitish-brown areas-plaques chagrinées -and one or two short pendulous polyps. The fundi appeared normal, and no " phakoma " could be detected in the retina. No other congenital malformation could be found by external examination, the skull and the palate were of normal shape, and the testicles completely descended; one observer, however, thought the patient's hands were ape-like. The kidneys could not be felt; a pyelogram was, unfortunately, not practicable. The blood urea was $38 \mathrm{mg}$. per 100 c.c.; laboratory investigations showed no abnormality in urine, blood, etc. There was no spasticity or paresis, and the reflexes were normal.

The most interesting phenomenon was a total irregularity of the pulse. On palpation it could not be decided with certainty whether this was due to fibrillation or to numerous extrasystoles, though there were periods when three to four beats in quick succession could be felt after each normal beat. It could not be ascertained whether this irregularity had been present for a long time or even since birth, but it was present during the past two years while the patient was under observation. There was no history of rheumatic fever or any other illness that might have affected the heart, and the patient never complained of shortness of breath or palpitation, nor was he cyanosed. The apex beat was in the fifth intercostal space in the 


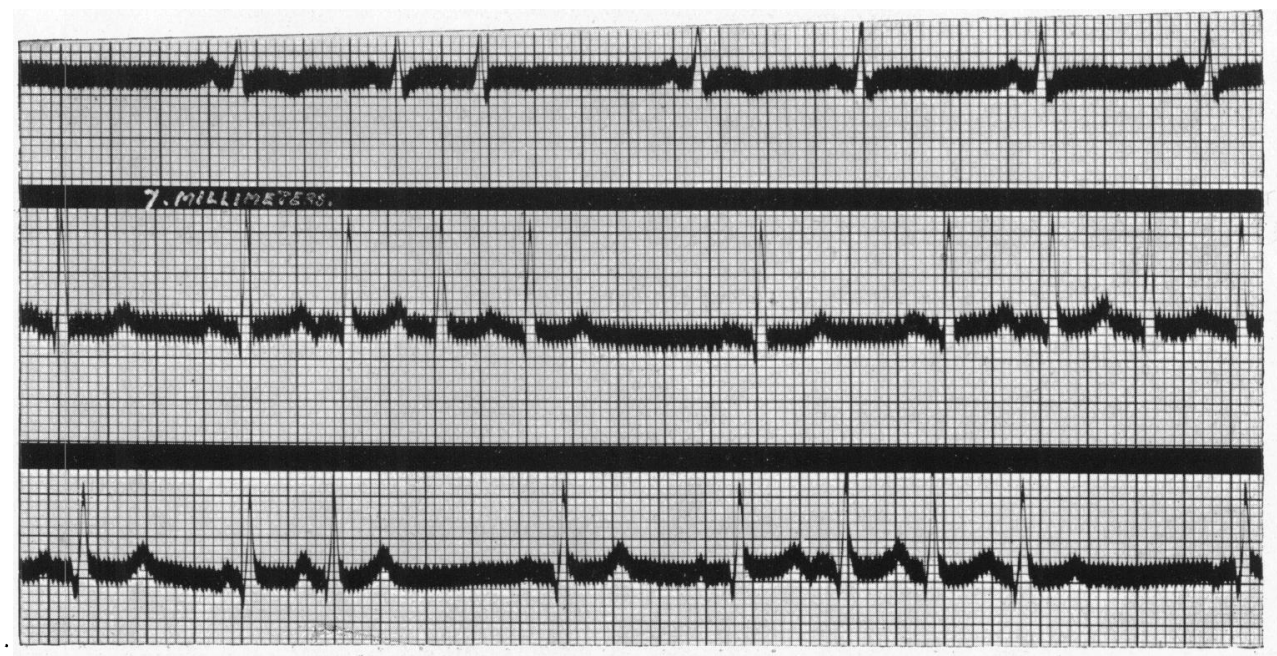

FIG. 1.-Electrocardiogram showing short paroxysms of supra-ventricular extrasystoles and some change of pace-maker shown in lead III.

midclavicular line ; the heart did not appear to be enlarged on percussion, no thrill could be felt, and no murmur was audible, but the action was quite irregular and seemed somewhat faster than at the periphery, presumably because some of the contractions were not powerful enough to reach the radial pulse. The blood pressure was $120 / 70$, the average pulse rate 85 . A cardiogram taken on 19/6/1944 (Fig. 1) showed paroxysms of extrasystoles of auricular origin, the'latter following each other in ${ }^{-}$such quick succession that their $\mathrm{P}$ waves were mostly

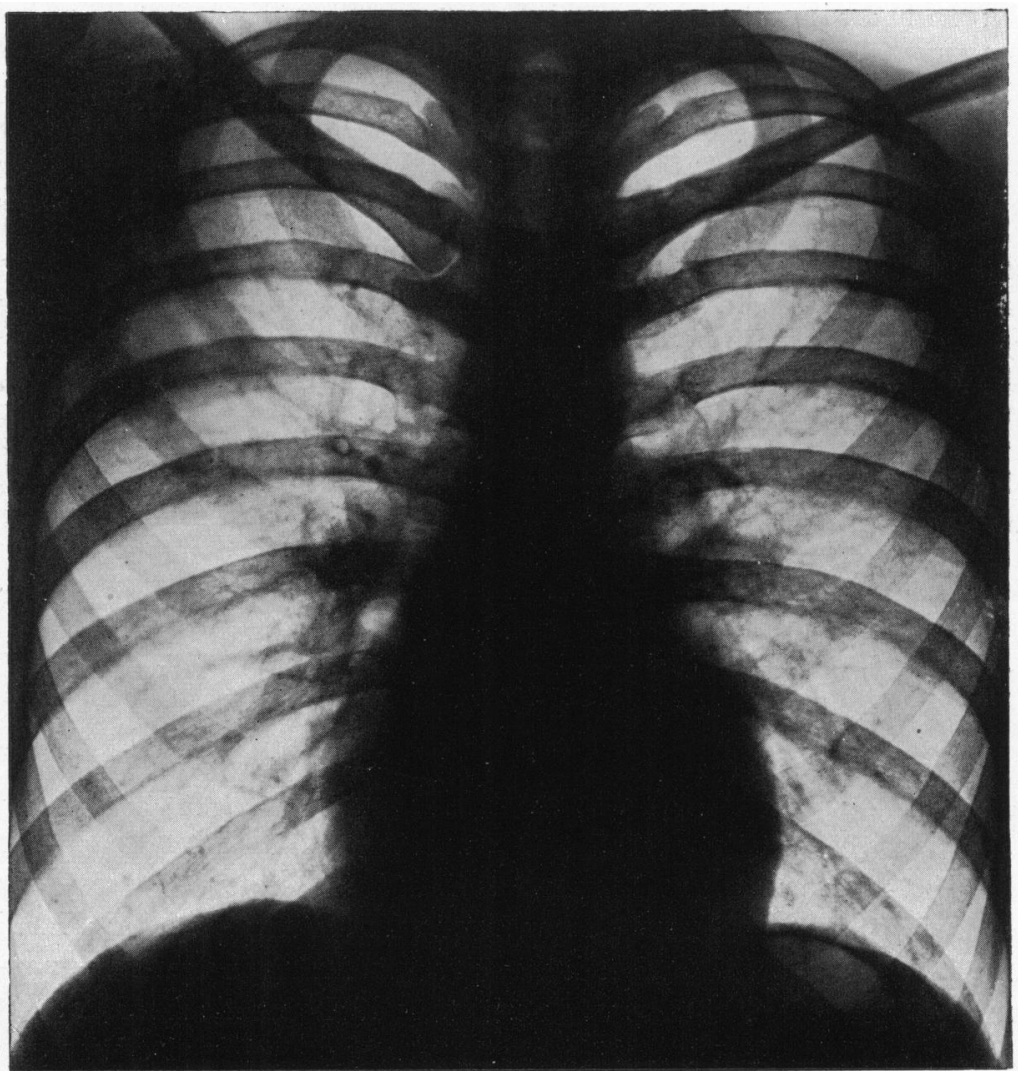

FIG. 2.-Teleradiogram, showing a globular heart with some enlargement to the right. 
superimposed on the $T$ waves of the preceeding beats. It appeared that most normal beats were a stimulus for a short chain of extrasystoles. Further evidence of myocardial involvement was the negative and absent $T$ waves in the first lead; the conduction time and the QRS complexes were normal; the S-T segments were hardly displaced; there was no axis deviation. A teleradiogram (Fig. 2) showed a heart of globular shape, the hypertrophy being mainly to the right, with some rounding of the apex; on screening in the left (II) oblique position, the left ventricle appeared closer to the spinal column than normal. This general hypertrophy may account for the balance of the electrical axis in the cardiogram. No evidence of enlargement of the pulmonary conus or arch or of the left auricle was seen on fluoroscopy; the aorta appeared in a normal position. The irregularity of the heart action was clearly seen. Bones and lungs showed no evidence in the X-ray of the osseous and pulmonary manifestations recently described by Ackermann (1944), who gives references to previous findings in these organs. The lungs were clear and the bones did not show cyst formation which has been found in some cases of tuberous sclerosis. These cysts in the bones, together with the skin changes, are a possible link with, von Recklinghausen's disease.

\section{Discussion}

Clinical signs of heart involvement due to rhabdomyomata are very unusual. In fact, since the first description of rhabdomyoma by von Recklinghausen in 1862 , there seems to exist only one report of a case with an electrocardiogram, taken because of tachycardia and arrhythmia, that was proved at necropsy to be caused by rhabdomyoma. This case, of Wegman and Egbert (1935), a ten months' old girl, the only child of young and healthy parents, had attacks of convulsions with cyanosis and pallor, and a pulse rate of over 200 with an irregular rhythm; periods of varying length of rapid, irregular beats were interrupted by short periods of stoppage or slowing. In the cardiogram reproduced by them, the rhythm is regular, however, and the tachycardia thought to be auricular flutter. There was no enlargement of the heart in the teleradiogram. A diagnosis of a congenital heart lesion was made, the nature of which was not clear. At the post-mortem examination rhabdomyoma nodules were found invading the conducting system, and also situated on the mitral and tricuspid valves. There were numerous small cysts in the kidneys. No evidence of tuberous sclerosis was found in the brain.

In the 51 cases of congenital rhabdomyoma of the heart that have been reported up to date, 29 (57 per cent.) were associated with tuberous sclerosis according to Labate (1939), who gives a tabulated survey of all cases. In 4 cases tuberous sclerosis was not found; in 18 it is not mentioned. Labate's own case was a cyanosed baby that lived only three hours: the heart was very enlarged, and had two large tumours in the interventricular septum, as well as various congenital malformations; tuberous sclerosis of the brain was present, and also a cerebral tumour on the floor of the left lateral ventricle.

No irregularity of the pulse was noted in the cyanosed baby reported by Rae (1938), but no cardiogram was taken; the heart appeared enlarged in the X-ray. At autopsy a large rhabdomyoma was found in the interventricular septum, as well as a patent foramen ovale and patent ductus arteriosus. There was also an enlarged liver and spleen. Rae himself comments that "it is unlikely that the conduction could have remained undisturbed."

Most of the cases reported were newborn babies or infants who lived a very short period. Five cases were over 15 years at the time of death; the oldest, that of Steinbiss (1923), was 35 years of age. Steinbiss reports on six cases that he has seen over a period of ten years; all of them had tuberous sclerosis and rhabdomyoma of the heart at post-mortem examination. He found that up to 1923, 30 cases of rhabdomyoma of the heart had been reported, of which 22 had tuberous sclerosis. Steinbiss sought especially in his cases of tuberous sclerosis for 
evidence during life of rhabdomyoma of the heart, but he could not find evidence of this in any of his cases by percussion and auscultation, and there was no alteration in the pulse.

It is indeed astonishing that clinical manifestations of rhabdomyomata have not been found more frequently. Whether the tumour appears as a solitary nodule, which may attain a considerable size, or scattered throughout the myocardium as the multiple type, or in form of diffuse rhabdomyomatosis, one would think that in most cases it would interfere with the spread of excitation within the heart or cause the formation of heterotopic beats. The effect will depend on its position; if the heart action is irregular, the tumour may be expected to lie in the path of the conducting system; sudden or early death may be due to obstruction of a valvular orifice. It is conceivable that large, solitary tumours protruding into a heart chamber might be made visible through contrast visualization after the method of Robb and Steinberg with diodrast.

As it is not uncommon to find ventricular tumours of the brain in tuberous sclerosis, it has to be borne in mind that the cardiac disturbance in our case might have its origin in some hypothalamic lesion.

\section{SUMMARY}

A case of tuberous sclerosis with an irregular heart action and cardiac hypertrophy is reported; it is assumed that this may be due to rhabdomyomata.

It seems that there are no published reports with cardiographic and $\mathrm{X}$-ray abnormalities of any cases, suspected during life of having rhabdomyoma.

I wish to thank Dr. T. Tennent, Medical Superintendent, St. Andrew's Hospital, Northampton, for his permission to give an account of the case.

\section{REFERENCES}

Ackermann, A. J. (1944). Amer. J. Roentgen., 51, 315.

Labate, J. S. (1939). Amer. J. Path., 15, 137.

Rae, M. V. (1938). Canad. med. Ass. J., 39, 63.

Steinbiss, W. (1923). Virchow's Arch., 243, 22.

Wegman, M. E., and Egbert, D. S. (1935). J. Pediat., 6, 818. 